
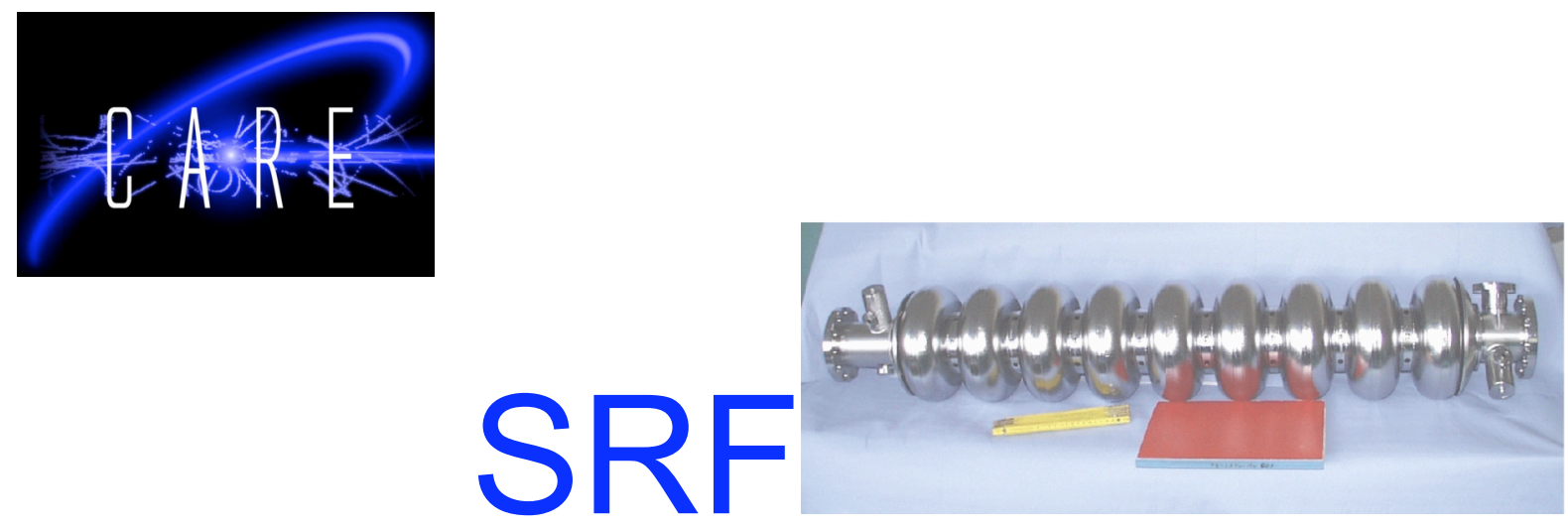

\title{
UHV Arc for High Quality Film Deposition
}

R. Russo, Istituto di Cibernetica "E.Caianiello" del CNR Pozzuoli (Na), and INFN-Na, Italy A. Cianchi, L. Catani, J. Lorkiewicz, INFN-Roma Tor Vergata, Italy

Y.H. Akhmadeev, N.N. Koval, Institute of High Current Electronics, HCEI, Tomsk, Russia

J. Langner, M. Sadowski, Soltan Institute for Nuclear Studies, SINS, Swierk, Poland

R. Polini, Dipartimento di Scienze e Tecnologie Chimiche, Università di Roma "Tor

Vergata", Italy

B. Ruggiero, Istituto di Cibernetica "E. Caianiello" del CNR, Pozzuoli (Na) Italy

S. Tazzari, Università di Roma "Tor Vergata" and INFN-Roma Tor Vergata, Italy

\begin{abstract}
The vacuum arc is a well-known technique for producing coatings with enhanced adhesion and film density. Many cathodic arc deposition systems are actually in use in industry and research. They all work under (high) vacuum conditions in which water vapour pressure is an important source of film contamination, especially in the pulsed arc mode of operation. Here we present a cathodic arc system working under ultra-high vacuum conditions (UHVCA). We have used for arc ignition a Nd-YAG pulsed laser focused on the cathode surface, which provides a reliable system and allows eliminating all possible sources of contaminants. We have proven that the arc technique produces bulk-like films suitable for superconducting applications. UHVCA has been used to produce ultra-pure niobium films with excellent structural and electrical properties at a deposition temperature lower than $100 \mathrm{oC}$. The UHVCA technique therefore opens up new perspectives for all applications requiring pure films and low deposition temperatures.
\end{abstract}

International Conference on Metallurgical Coatings And Thin Films, ICMCTF 2006 May 1-5, 2006, San Diego, California, USA

Work supported by the European Community-Research Infrastructure Activity under the FP6 "Structuring the European Research Area" programme (CARE, contract number RII3-CT2003-506395) 
Keywords: Vacuum Arc, UHVCA, Thin Film, Superconductivity, UHV, Niobium

\section{Introduction}

Vacuum arc is one of the oldest techniques used for thin film deposition [1]. It presents several advantages over other techniques, mainly the ionized state of the evaporated material and the high ion energy and it produces coating with an enhanced adhesion and film density [2]. The main drawback is represented by the production of macroparticles on the cathode surface that become embedded in the film increasing its surface roughness. In recent year important progresses have been made in magnetic filtering macroparticle allowing the deposition of defect free films [3] and opening the road to new applications where a flat surface is needed. To increase the ionization state and to reduce the thermal load on cathode and substrate several groups developed and used the pulsed vacuum arc [4]. Very recently much progress has been made in understanding the nature of the cathode spot, the distribution of energy ions, the role of cohesive energy [5], the importance of atomic scale heating [6] and selfsputtering [7]. However oxygen and hydrogen incorporation in films produced in the pulsed mode [8,9] and in DC operation [10] is also often reported. It is believed the contamination originates from the water vapor layer that forms on the cathode surface between arc pulses when working in high vacuum, due to the residual water vapor present in the chamber, or from backstreaming of diffusion pump oil in case of DC operation [10]. The problem of water contamination can be particularly important in the case of deposition of materials with getter properties, like Niobium, Titanium, Vanadium, Zirconium. In particular the superconducting critical temperature $\left(\mathrm{T}_{\mathrm{c}}\right)$ of niobium is very sensitive to impurities and stresses. Very small amount of oxygen can lower the $T_{c}$ value [11], while a compressive stress can increase the transition temperature $[12,13]$. The contamination due to vacuum conditions 
has probably been one of the main reasons for the very limited applications of the arc technique to the production of superconducting elements.

A cathodic arc source working in UHV (UHVCA) can solve the problem of contaminations, opening the road to applications where very pure metallic films are needed.

Another important source of contaminants is due to memory effects of different cathode materials and it was studied by D.R.Martins [14]. The energetic neutrals, ions and macroparticles, can produce sputtering from the chamber walls coated with the previous cathode element. To prevent this kind of contamination we devoted our main vacuum systems to the investigation of deposition of high purity niobium film only, and used a separate system to deposit other materials less sensitive to impurities (such as tungsten and nitrides).

We designed, constructed and put into operation a UHVCA to produce very high quality niobium films for application to accelerating niobium coated superconducting copper cavities [15], with the aim to improve their performance at accelerating fields higher than $15 \mathrm{MV} / \mathrm{m}$ compared to that of cavities currently produced by cylindrical magnetron sputtering [16].

\section{Experimental-Set-up: Description and Results}

The schematic drawings of our UHVCA systems are shown in Fig.1. The conical cathode (usually $25 \mathrm{~mm}$ high) is obtained from a $50 \mathrm{~mm}$ diameter high purity Niobium rod and fastened to a water-cooled $\mathrm{Cu}$ support. An In-Ga eutectic provides enough thermal contact between the cooled copper and the niobium cathode to prevent cathode overheating for DC arc currents up to 140A. The vacuum chamber around the $\mathrm{Nb}$ cathode, also conical and water cooled, with a final inner diameter of $90 \mathrm{~mm}$, was fabricated from a single stainless steel rod and works as the anode. The UHVCA is mounted inside a UHV chamber pumped down by an oil-free pumping system consisting of a membrane pump on the foreline and a drag turbo molecular pump. The system is equipped with a Residual Gas Analyzer (RGA) to monitor the presence of contaminants before and during the coating. The base pressure of $1-2 \times 10^{-8} \mathrm{~Pa}(1-$ $2 \times 10^{-10}$ mbar) in the system is reached after one night bake at $150^{\circ} \mathrm{C}$. The ignition of the arc 
discharge in this region of pressure can be a problem, and a conventional surface ceramic trigger was found not reliable. Moreover evaporation from a surface ceramic trigger was found to contaminate the metallic film particularly when the arc was unstable and several ignitions where needed for a single deposition. We tested several triggering systems to ignite the arc discharge and the final adopted solution is a compact Nd-YAG Laser focused on the cathode surface that, in our experience, is the cleanest and most reliable way of triggering in UHV [17]. The main coil surrounding the anode chamber generates the magnetic field needed to confine the arc spot to the cathode surface on which the hot spot moves with a measured speed of about $10 \mathrm{~m} / \mathrm{s}$. Typically, it generates a magnetic field of about 150 Gauss on the cathode surface. A niobium floating potential electrode surrounds the cathode to prevent the discharge from moving downwards, towards the bottom part of the chamber.

In the unfiltered configuration the copper and sapphire substrates to be coated are secured to a massive $\mathrm{Cu}$ sample-holder placed in front of the cathode at a distance of about $50 \mathrm{~cm}$ from it, electrically insulated from the vacuum chamber. An electrically insulated rotating shutter allows to expose one sample at the time to the plasma beam, making possible the deposition of several samples in different conditions without breaking the vacuum. In this configuration samples face the cathode and macroparticles can be embedded in the growing film. While not contaminating the film, macroparticles increase its surface roughness and in RF cavities applications may become sources of field emission. To reduce this effect a magnetic macroparticle filter has been built and studied. The filter coil arrangement was designed to force electrons to follow magnetic field lines with orbit radii smaller than the vacuum chamber elbow one. Samples are mounted on a sample holder placed $30 \mathrm{~cm}$ downstream from the $90^{\circ}$ elbow (see Fig. 1 b). A magnetic field of about 70 Gauss was used to guide the plasma trough the duct. In both systems the lowest possible arc current for stable DC operation was about $60 \mathrm{~A}$ while we were limited by our cooling system to a maximum current of $140 \mathrm{~A}$ in the continuous mode (at higher currents the In-Ga eutectic diffuses into copper and niobium 
reducing the thermal contact between the niobium cathode and the water cooled copper support ).

To improve and control the deposition rate in the filtered and unfiltered systems two additional Helmholtz coils have been wound around the respective main chambers (see Fig.1). They generate a magnetic field that guides the plasma to the sample-holder and increases the deposition rate by up to $40 \%$ in the unfiltered system (see Fig.2a). The sample holder is biased to a negative constant voltage in order to reflect electrons and to collect only ions, avoiding substrate heating due to the large electron current and keeping the sample holder temperature below $100^{\circ} \mathrm{C}$.

Since niobium ions have kinetic energy higher than $100 \mathrm{eV}$ and are multiply charged (mean ionization for niobium +3 [18], the average kinetic energy of niobium atoms reaching the substrate is estimated to be about $250 \mathrm{eV}$ for a typical $-40 \mathrm{~V}$ bias (about $350 \mathrm{eV}$ for $-80 \mathrm{~V}$ bias), large enough to produce some sputtering from the growing niobium films [7]. The ionization state +3 also provides an additional potential energy of about $46 \mathrm{eV}$ [6] that is released on the substrate when ions are neutralized. The shutter can be either electrically connected to the samples or left floating: in the latter case its average voltage is about $-20 \mathrm{~V}(-40 \mathrm{~V})$ in the unfiltered (filtered) system.

The ion current recorded on the sample holder fluctuates due to the explosive nature of the cathode spot: its average value is found to saturate for a voltage bias higher than $-40 \mathrm{~V}(-80 \mathrm{~V})$ in the unfiltered (filtered) system and to increase linearly with arc current (see inset Fig.2b). The ion current of both systems, averaged on 100 acquisitions, is plotted in Fig. 2 as a function of the current in the additional helmholtz coils: the error bars represent the standard deviation of the average ion current values recorded during the 100 acquisitions. In the unfiltered system (Fig.2a) the niobium ion current (full squares) increases with the additional coil current reaching a maximum at about 15A. A simultaneous reduction in the amplitude of ion current fluctuations during deposition has also been observed, the amplitude reaching a 
minimum at a coil current of $20 \mathrm{~A}$, corresponding to a magnetic field of about $1.5 \mathrm{mT}$ at the coil center. The figure is very similar for different arc currents, and for different cathode materials. In particular Tungsten has also been studied in a separated chamber, (open circle in Fig 2a): the presence of the maximum in the average ion current is unchanged, whereas the current fluctuations are reduced at higher magnetic fields.

In the unfiltered UHVCA system the maximum in the average ion current is attributed to an optimised magnetic field configuration for the plasma transport [18], whereas the minimum in the current fluctuations around $20 \mathrm{~A}$ in the additional coils needs further investigations.

In the filtered system (Fig.2b) the magnetic field configurations and the plasma transport efficiency improve dramatically when additional coils are used. A maximum average ion current of $400 \mathrm{~mA}$ is obtained for an arc current of about $120 \mathrm{~A}$ with a current of about $100 \mathrm{~A}$ in the additional coils, followed by some saturation. In the best configuration we obtained a deposition rate of about $10 \mathrm{~nm} / \mathrm{s}$ in the unfiltered system and $1 \mathrm{~nm} / \mathrm{s}$ in the filtered system.

The efficiency of the filtered system was not completely optimized since it is known that a positive bias on the filter can improve the plasma transport in the duct [3]. Since it is quite difficult and expensive to electrically insulate the magnetic filter in UHV, the possibility to add a biased electrode on the outer wall on the duct [19] and improved filter design to improve filter transparency are under study.

The base pressure is in the $10^{-8} \mathrm{~Pa}\left(10^{-10} \mathrm{mbar}\right)$ range, but, as soon as the arc discharge starts, the total pressure increases up to $10^{-4} \mathrm{~Pa}\left(10^{-6} \mathrm{mbar}\right)$ and is independent on the system configuration (filtered or unfiltered). When $\mathrm{Nb}$ starts being evaporated from the cathode and the chamber walls become coated with a fresh $\mathrm{Nb}$ film, outgassing from surfaces decreases rapidly and stays almost stable during deposition. After one minute operation the contaminants other than hydrogen fall below the detection limit of a Quadrupole Mass Analyzer (usually $10^{-6} \mathrm{~Pa}$ ) and can only be detected using a Residual Gas Analyzer. During the DC arc operation the residual gas is almost exclusively hydrogen, its partial pressure being 
usually $1-2 \times 10^{-4} \mathrm{~Pa}$, more than 3 orders of magnitude higher than that of other contaminants which are usually in $10^{-7} \mathrm{~Pa}$ range or below. This excess of hydrogen is believed to be generated by the $\mathrm{Nb}$ bulk cathode providing an essentially 'infinite' source of this gas. The hydrogen partial pressure can be further reduced by reducing the cathode hydrogen content by outgassing it at high temperature in UHV before mounting.

All other gases are present in small quantities only on the chamber wall surface, which makes their partial pressures drop after only one minute of operation. Usually a first sample location is used only to completely clean the system while on the remaining 5 positions samples are mounted and coated under the chosen conditions.

\section{Results on niobium films}

One of the main parameters to check the quality of niobium film is the Residual Resistivity Ratio (RRR defined as the Resistivity at room temperature divided the Resistivity at 10K) since it is very sensitive to impurity. Typical RRR values for $\mathrm{Nb}$ films deposited by sputtering at room temperature range from 2 to 10 , while niobium bulk usually ranges from 40 to 500 .

Our films RRR values are always higher than 20 and in some cases values larger than 50 have been recorded on samples deposited at room temperature. Note that similar values, strong indications of very pure films, can also be obtained by sputtering but only by heating the substrate to high temperature (about 400C). The impurity content of our samples has been checked by standard spectroscopy analysis (Auger, XPS, EDX) : once the surface oxide layer has been removed, contaminants are usually below the detection limits. SIMS analysis are under way to check the presence of impurities at lower levels.

The $\mathrm{Nb}$ film structure has been analyzed using X-ray diffraction and atomic force microscopy, while morphology was investigated by Field Emission Gun Scanning Electron Microscopy (FEG-SEM, Leo Supra 35) images. X-ray diffraction (XRD, Philips X'Pert Pro) pattern (see Fig.3) are collected using graphite filtered $\mathrm{Cu}-\mathrm{K}_{\alpha}$ radiation, in the $\theta-2 \theta$ configuration. Due to a relatively small film thickness X-rays are not all stopped in the 
niobium film but also penetrate the substrate. When the substrate is copper they produce fluorescence, thus increasing the background noise compared to the sapphire substrate. The position of the maximum in the diffraction peak is obtained by fitting the curve with an appropriate peak function (see inset of Fig.3b). The lattice parameter is then obtained by extrapolating the lattice parameter values calculated at various different angles to $2 \theta=\pi$. The results range from 0.3306 to $0.3318 \mathrm{~nm}$, indicating a much lower stress than observed in niobium deposited by magnetron sputtering $[12,13]$, a result also confirmed by inductive $T_{c}$ measurements performed on the same samples (see below). The width of the diffraction peak is also narrower than in the sputtering case, an indication of larger and/or more ordered grains. This result was also confirmed by Atomic Force Microscope pictures showing an average niobium grain size of 200nm (see Fig.4), to be compared to grain size of $80-120 \mathrm{~nm}$ usually measured on niobium film grown by magnetron sputtering.

The superconducting critical temperatures $T_{c}$ have been measured using an inductive technique described elsewhere [20]. Results range from $9.20 \mathrm{~K}$ to $9.32 \mathrm{~K}$ with a transition width always less than $0.02 \mathrm{~K}$, in good agreement with $\mathrm{Nb}$ bulk data $\left(\mathrm{T}_{\mathrm{c}}=9.26 \mathrm{~K} \Delta \mathrm{T}_{\mathrm{c}} \sim\right.$ $0.01 \mathrm{~K}$ ); a few typical transitions are shown in Fig.5. During transition a voltage signal is recorded while no voltage is seen elsewhere. No major differences are observed between the filtered and unfiltered cases, except that in the unfiltered case, when the macroparticles cover a non negligible (larger than $10 \%$ ) portion of the film surface the inductive measurement allowed to observe the transition of both film and macroparticles, as shown in Fig.5 (open circles). Differences in film critical temperature with respect to bulk value are much less than in the magnetron sputtering case where $T_{c}$ can become much lower (even below $4.2 \mathrm{~K}$ ) due to contamination [11] or higher (up to 9.5-9.6K) due to internal stress [12,13], indicating that our films are cleaner and less stressed than the typical magnetron sputtered films.

The roughness of $\mathrm{Nb}$ samples deposited on copper (measured using a mechanical alpha-step) is comparable to that of the copper substrate itself, whereas that of niobium films on sapphire 
is much smaller as shown by FEG-SEM micrographs of Fig.6. This clearly indicates that, on copper, the film roughness is determined by the substrate. When using a magnetic filter to reduce microdroplets the roughness of niobium film on sapphire is observed to be of the order of few nm, comparable to that of niobium sputtered films deposited on the same substrate.

\section{Conclusions}

We designed, built and characterized an arc source working in UHV conditions. (UHVCA) and used it to produce very high quality superconducting niobium films at room temperature. The superconducting properties are very similar to those of bulk material and lattice parameter show the stress is lower than in niobium sputtered films. The UHVCA system has shown excellent reproducibility and reliability also thanks to laser triggering. The system is ultraclean and after conditioning for about 1 minute no contaminant can be detected by a QMA. The total pressure during arc operation is in the $10^{-5} \mathrm{~Pa}$ range $\left(10^{-7} \mathrm{mbar}\right)$ and consists mainly of hydrogen degassed from the niobium cathode. A filtered arc was used to produce samples on which no droplets are detected of a size exceeding the sensitivity limit of the instrument used, the film superconducting and structural properties remaining unchanged. To our knowledge this is the first vacuum arc working in UHV; it opens very interesting perspectives for the deposition of very clean and pure metallic films and for applications where contaminations have to be minimized.

\section{Acknowledgement}

We acknowledge the support of the European Community-Research Infrastructure Activity under the FP6 "Structuring the European Research Area" programme (CARE, contract number RII3-CT-2003-506395). 


\section{Reference}

[1]André Anders, IEEE Trans On Plasma Science, 31, (2003) 1060

[2]R.L. Boxman, P.J. Martins, D.M. Sanders Handbook of vacuum arc science and technology, Noyes, Park Ridge, NJ, 1995

[3] A. Anders, Vacuum 67 (2002) 673-686, D.M. Sanders, A. Anders, Surf. Coat. and Tech. 133-134 (2000) 78 R.L. Boxman et al. Surf. Coat. and Tech. 86-87 (1996) 243

A. Anders, Surf. Coat. and Tech. 120-121 (1999) 319

[4] T. Witke et al.rSurface and Coatings Technology 126(2000)81

[5] André Anders, Appl. Phys. Lett., 78 (2001) 2837

[6] André Anders, Appl. Phys. Lett., 80, (2002) 1100

[7] André Anders, Appl. Phys. Lett. 85, (2004) 6137

[8] J.M. Schneider, et al., Appl. Phys. Lett. 74(1999)200

[9] D.M. Sanders, A. Anders Surf. Coat. and Tech. 133-134(2000)78

[10] V.I. Gorokhovsky et al. Surf. Coat. Technol. 140 (2001) 215

[11] J.P. Carbotte, Rev. Mod. Phys. 62 (1990)1027 and references therein

[12] G. Heim, E. Kay, J. Appl. Phys. 46 (1975) 4006

[13] R. Russo, S. Sgobba, Part. Accel., 60 (1998) 135

[14] D.R. Martins, M.C. Salvadori, P. Verdonck, I.G. Brown, Appl. Phys. Lett. 81(2002)1969

[15] H. Padamsee, Supercond. Sci. Technol. 14 (2001) R28-R51

[16] C. Benvenuti et al., PhysicaC 351 (2001) 421

[17] J. Langner et al., Proc. International Symp. PLASMA-2003 on "Research and Application of Plasmas", Warsaw (Poland) 2003

[18] A. Anders and G. Y. Yushkov, J. Appl. Phys., 91 (2002) 4824

[19] Zhang et al., Rev. Sci. Instrum., 70, (1999) 3329

[20] R.Russo, L. Catani, A. Cianchi, J. Langner, and S. Tazzari, Supercond. Sci. Technol., 18 (2005) L41-L44 


\section{Figure caption}

Figure 1 a) Schematic drawing of the UHVCA system. The cathode(1) is mounted on a water cooled copper support (2) and inserted in a water cooled stainless steel anode(3). A laser beam (4) provides the arc ignition and a niobium floating potential electrode prevents discharge going in the bottom part of the system. The magnetic coil (5) confines the hot spot on the flat cathode surface, whereas two additional magnetic Helmholtz coil (6) improves deposition rate control on the sample holder (7). A rotating shutter (8) allows deposition of one sample at time without exposing the other substrates to the plasma beam: both, shutter and sample holders are electrically insulated from the main chamber (9). The CF100 pumping port (10) is located on the main chamber. b) Schematic drawing of the UHVCA system with a magnetic filter for macroparticles. Coils (11) are designed to guide electrons through the duct, while heavy macroparticles hint and stick on the duct walls. A copper disc (12) can be used to support samples (7) in different locations (can be also used in unfiltered system)

Figure 2 Ion current on the sample holder as function of the current in the additional coils for filtered and unfiltered system: a) unfiltered system, Arc current $80 \mathrm{~A}$, Voltage bias $-40 \mathrm{~V}$, b) filtered system Arc current $140 \mathrm{~A}$, bias $-80 \mathrm{~V}, \mathrm{I}_{\text {filter }}=2.5 \mathrm{~A}$ The Data points are the average values on 100 acquisitions, the error bar indicate the standard deviation calculated on 100 data points. The inset shows the niobium ion current in the unfiltered system as function of the arc current with zero current in the additional coils: the black line is a linear best fit.

Figure 3 a) X-ray spectra of Niobium films on copper and on sapphire as recorded as function of $2 \theta$ in degree. The upper curve is relative to $\mathrm{Nb}$ film growth on copper: the high background signal is due to fluorescence. Niobium films growth on both substrate preferentially along the 
(110) direction, but other orientations, indicated by the $(h k l)$ notation in the figure, are also present. The inset show the details of a (211) niobium peak on sapphire substrate with its best fit. The $\theta$ position of the maximum value of the peak for lattice parameter calculation is obtained from the fit.

Figure 4 AFM image of a niobium film deposited on sapphire (thickness 2 micron, $I_{\text {arc }}=120 \mathrm{~A}$, $V_{\text {bias }}=-40 \mathrm{~V}$ ): the average niobium grain dimension is about $200 \mathrm{~nm}$.

Figure 5 Inductive $T_{c}$ measurement for typical niobium samples produced by UHVCA in filtered (full black) and unfiltered system (open circle). The sharp transition width $(<0.02 \mathrm{~K})$ is similar to bulk and it is strong indication of uniform and clean films. The open circle is a measurement of a sample with macroparticles covering a large fraction of the surface. Two transition are visible: the first due to the film and the second due to macroparticles. In both case transition widths are about $0.01 \mathrm{~K}$ for film and for macroparticles.

Figure 6 a) FEG-SEM micrographs of a niobium film deposited on copper in the unfilter system. Arrow indicates some of macroparticles present on the surface, however, the roughness is mainly due to the copper substrate. b) FEG-SEM micrographs of a niobium film deposited on sapphire in the filter system. The surface is very flat and no macroparticle are visible. c) detail of the morphology for a 200nm thick niobium film deposited on sapphire. 
(9)

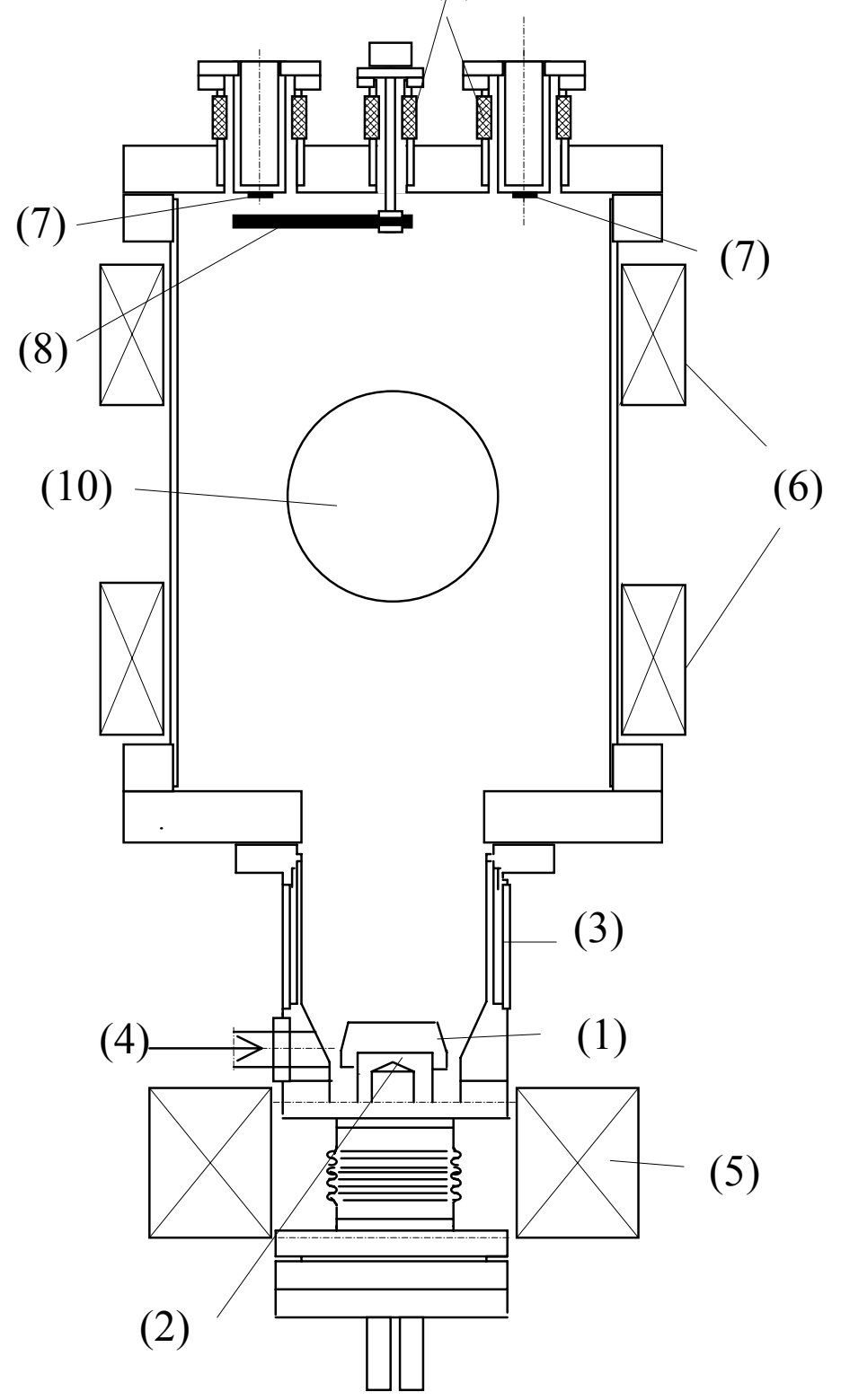

Figure 1a 


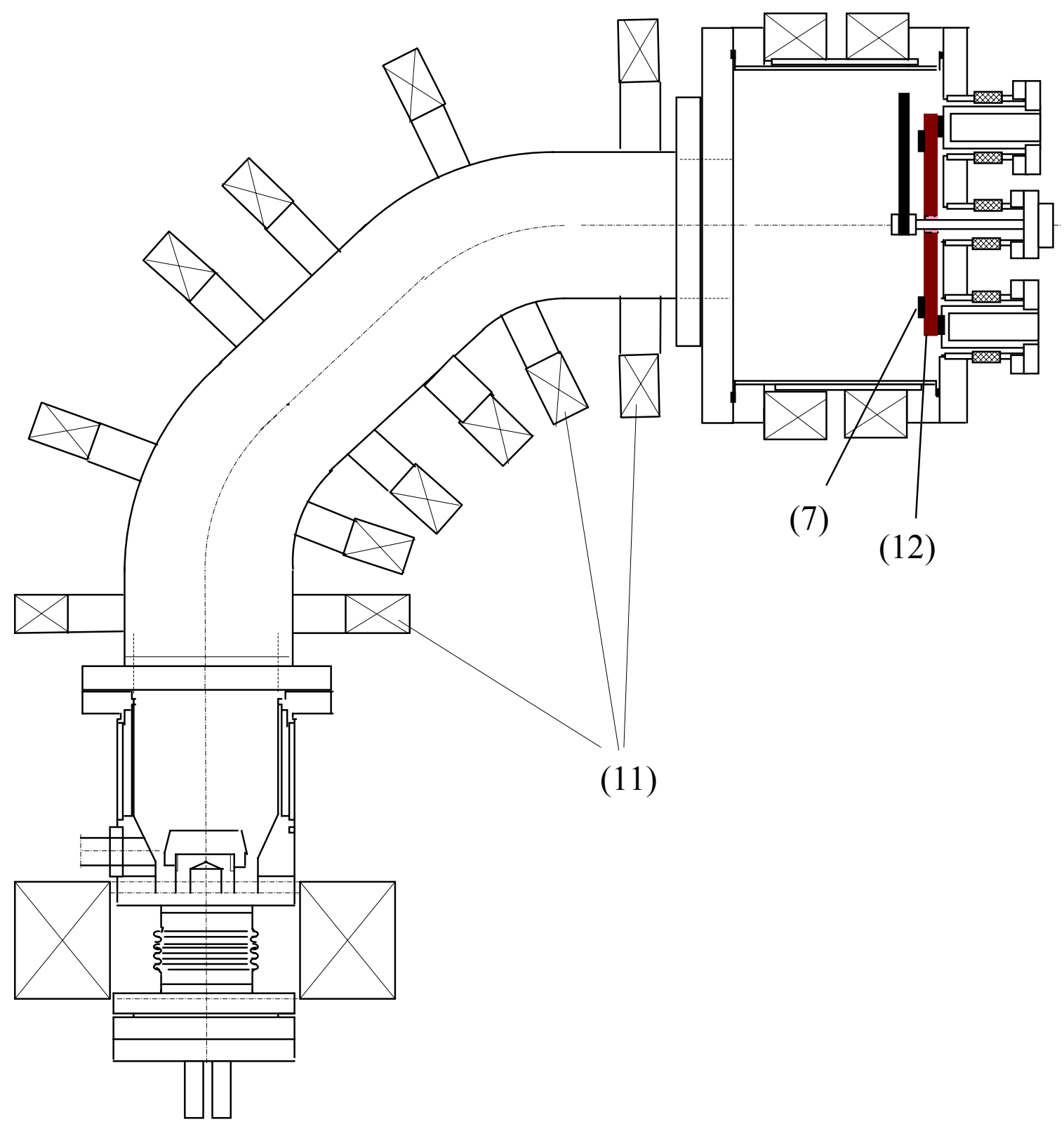

Figure $1 b$ 


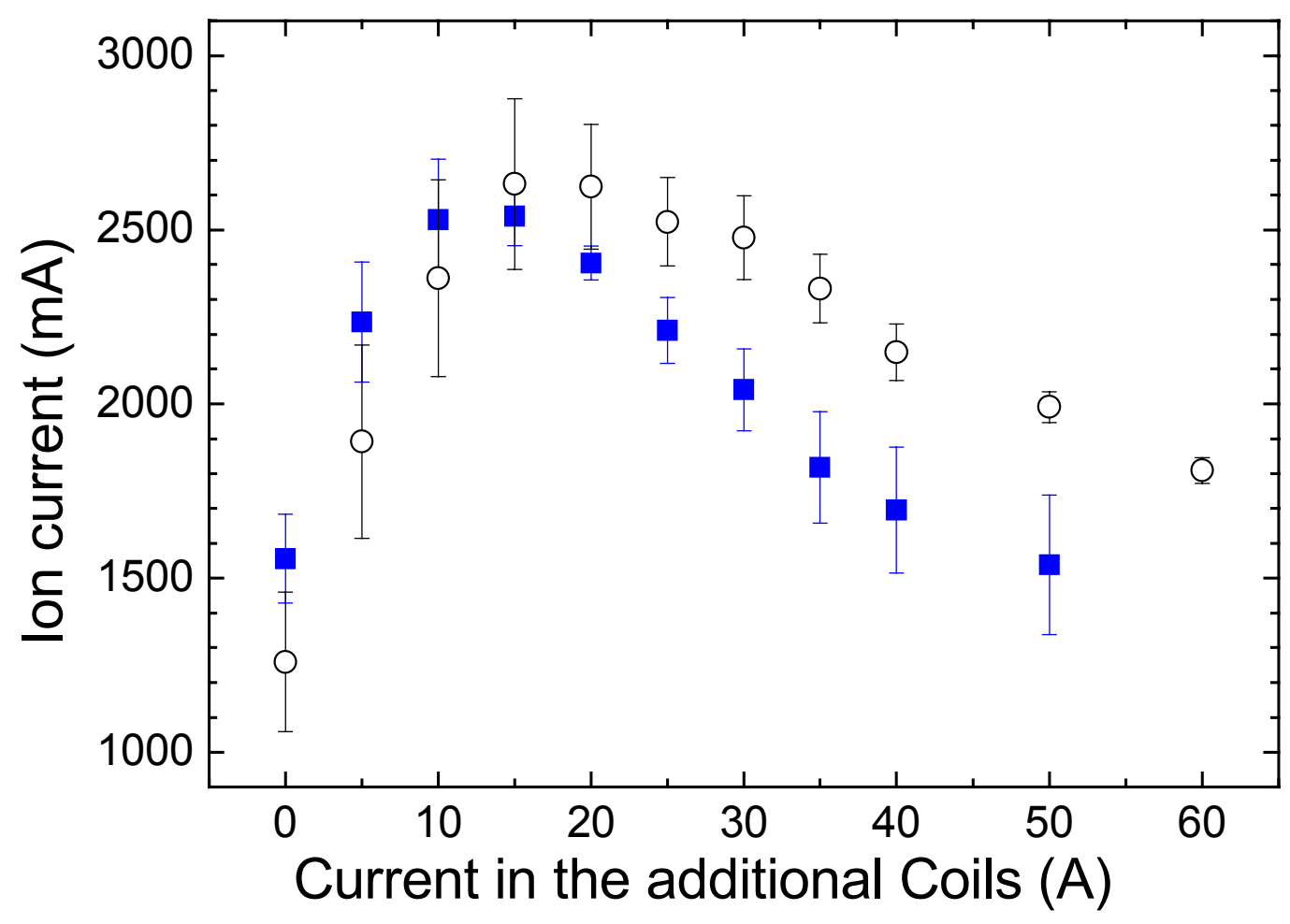

Figure 2a 


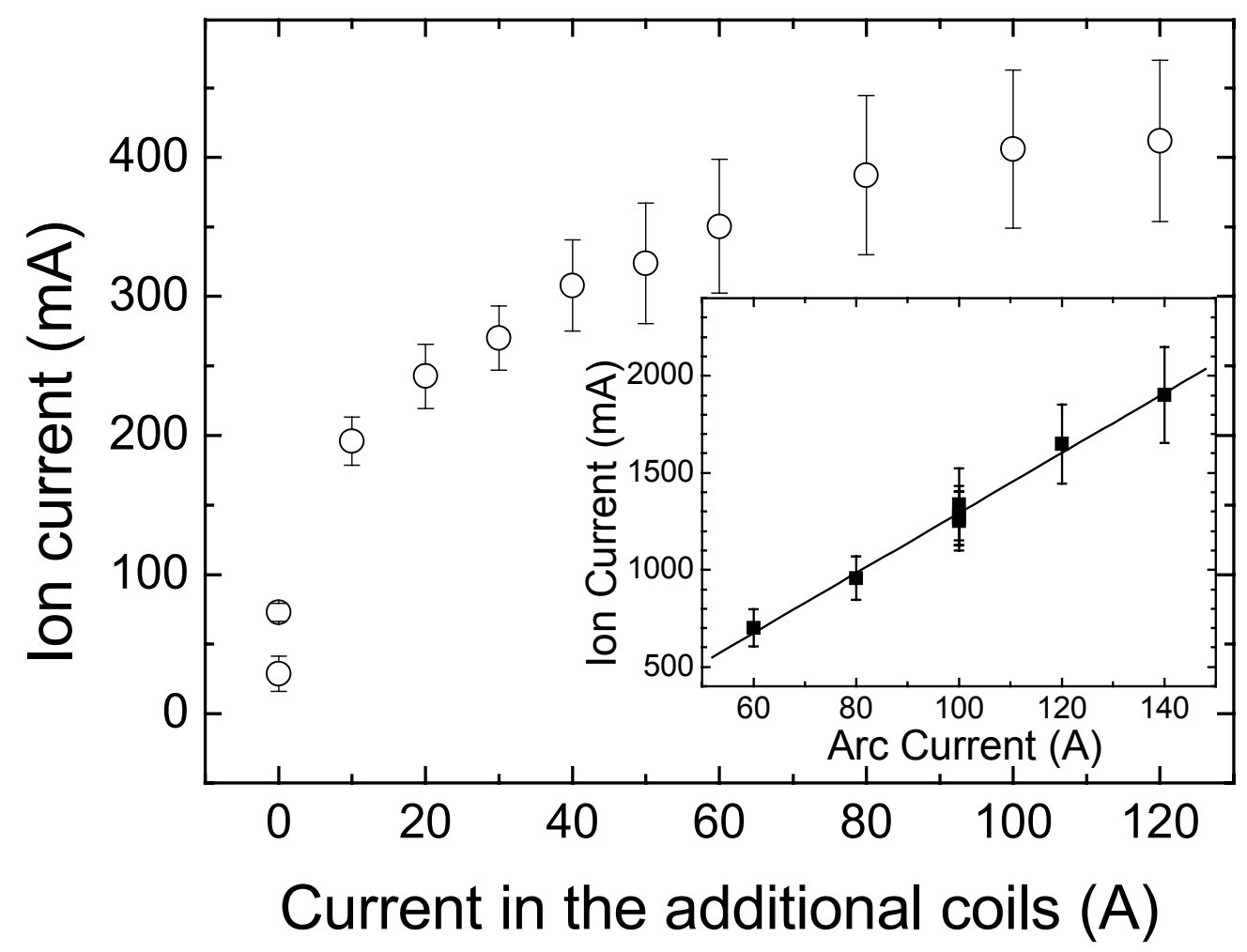

Figure $2 b$ 


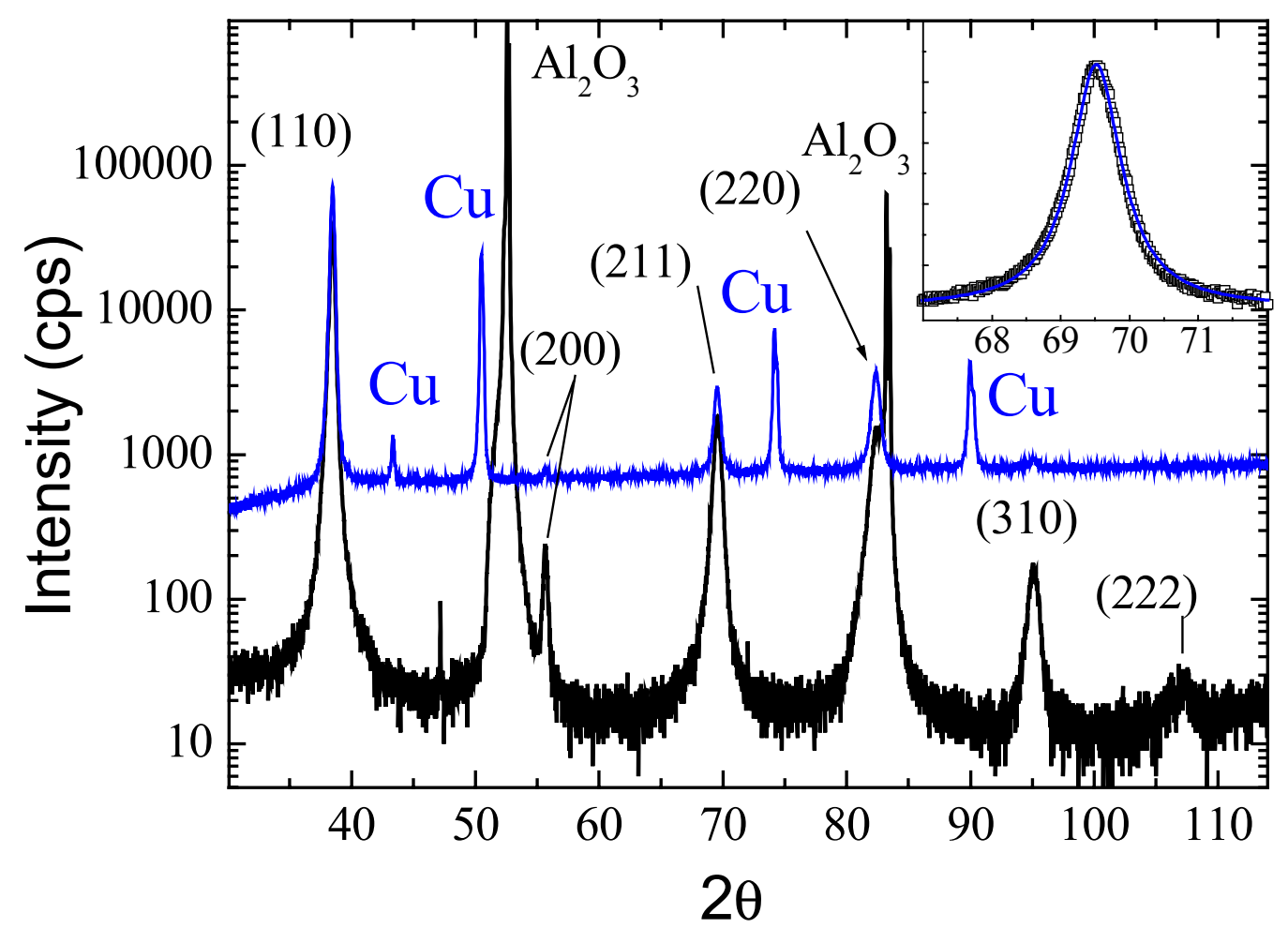

Figure 3 


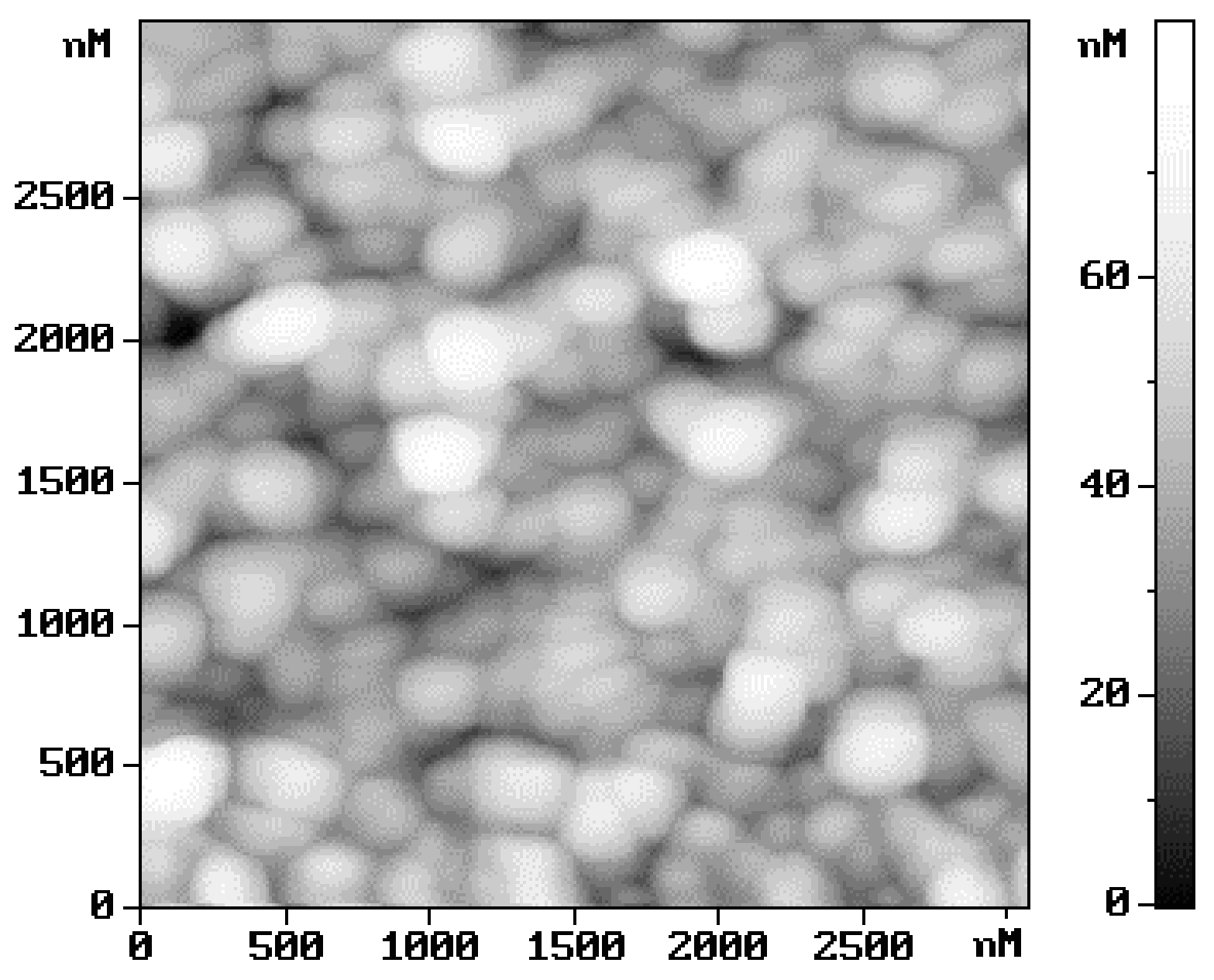

Figure 4 


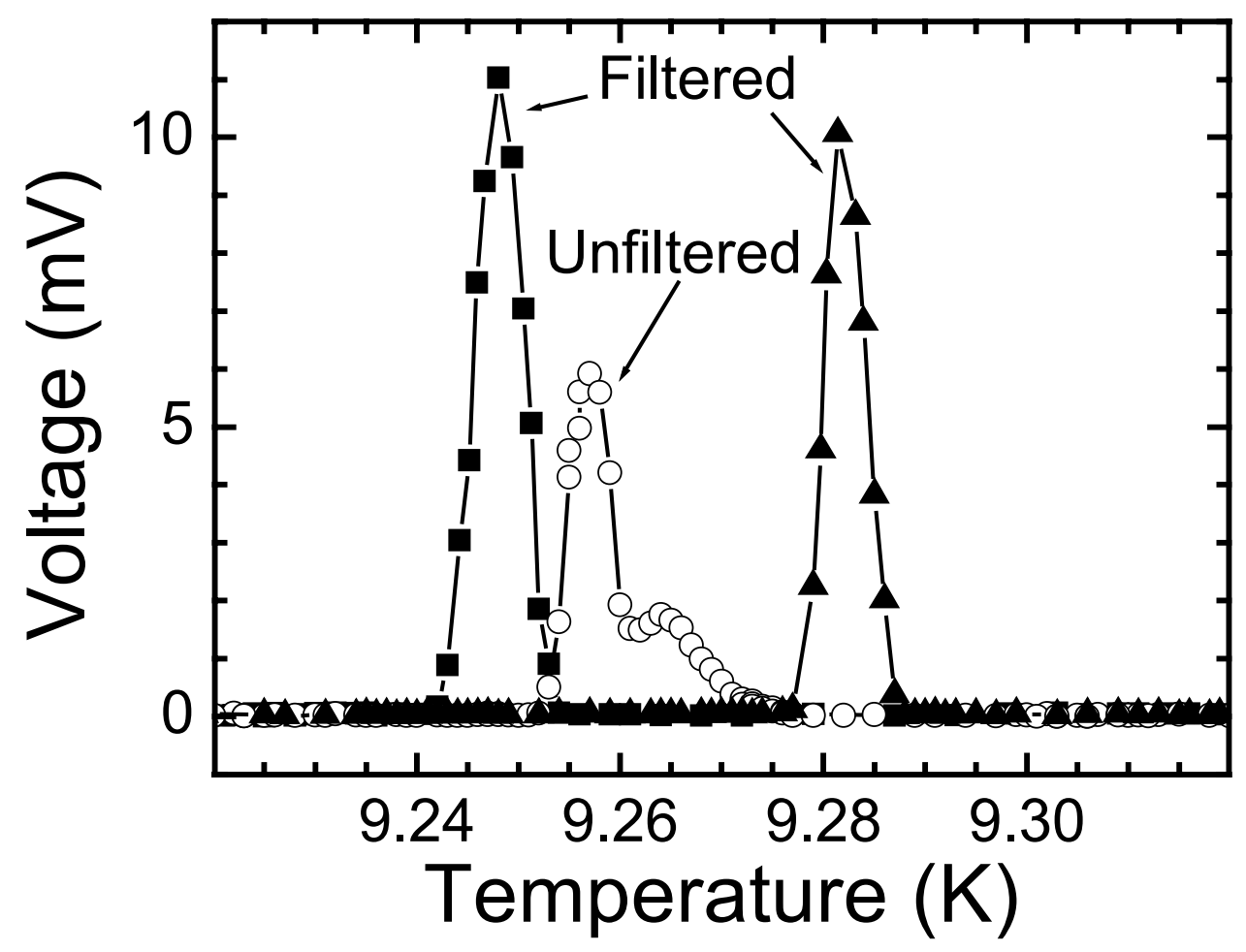

Figure 5 


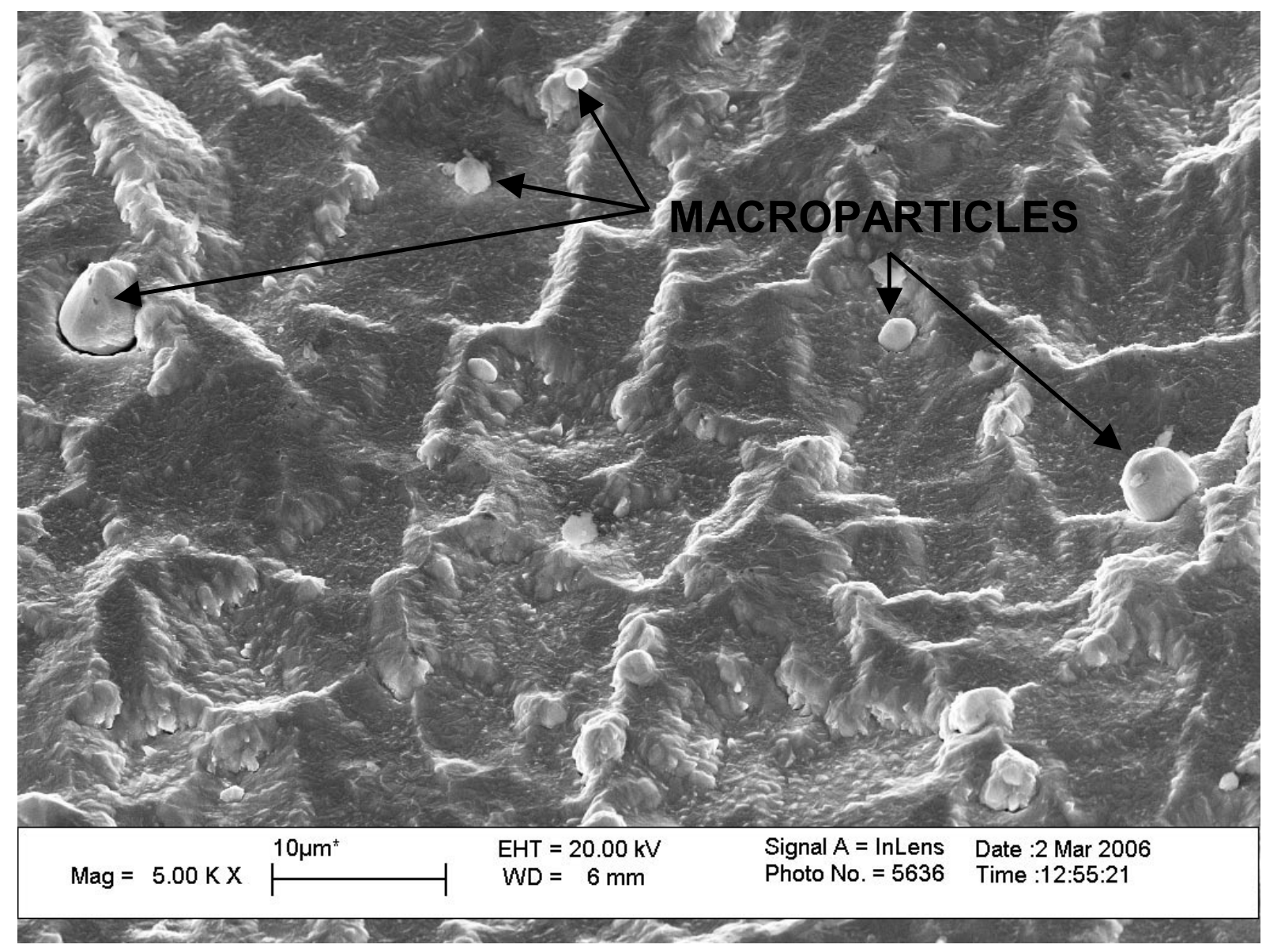

Figure 6a 


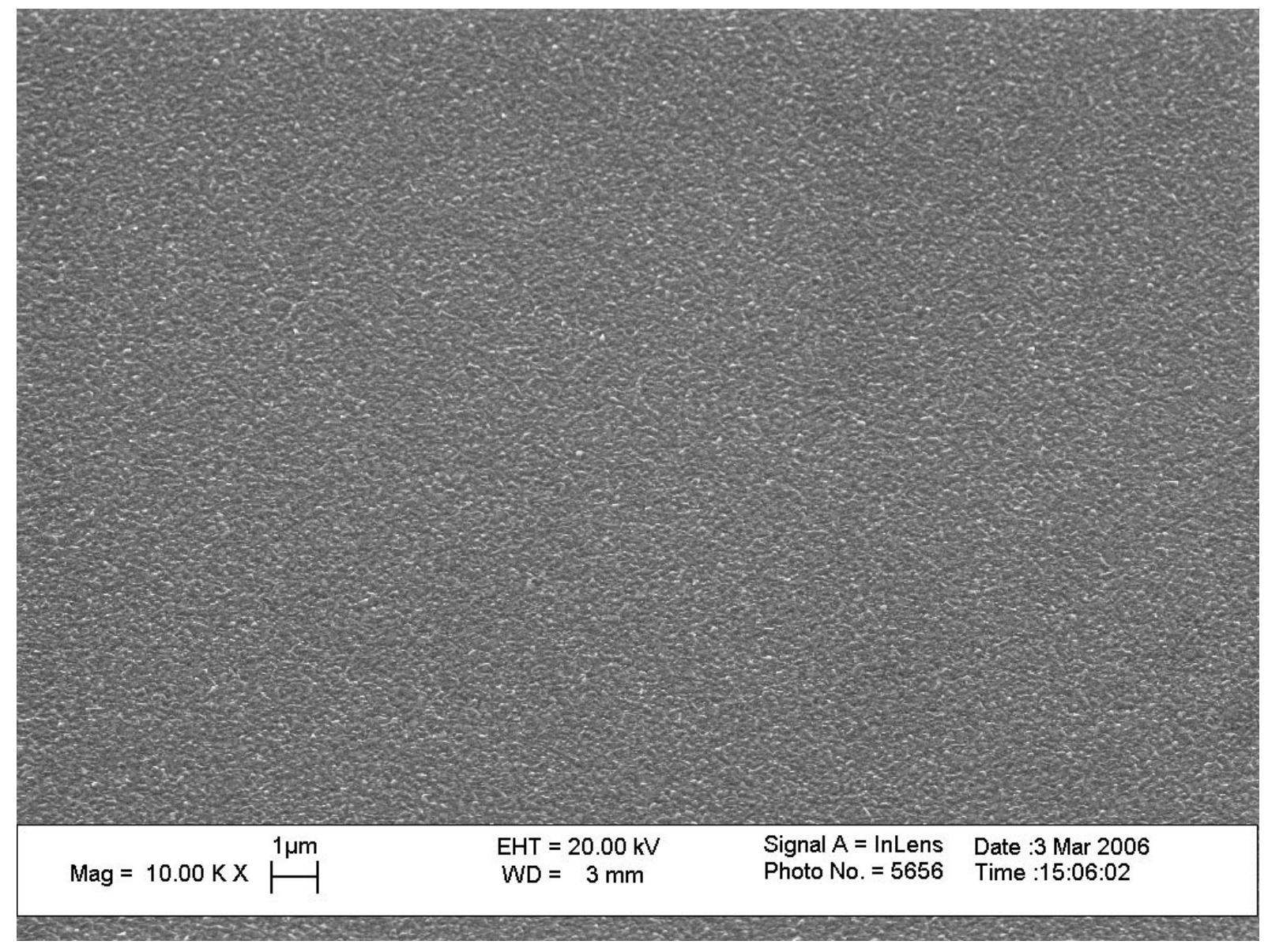

Figure $6 b$ 


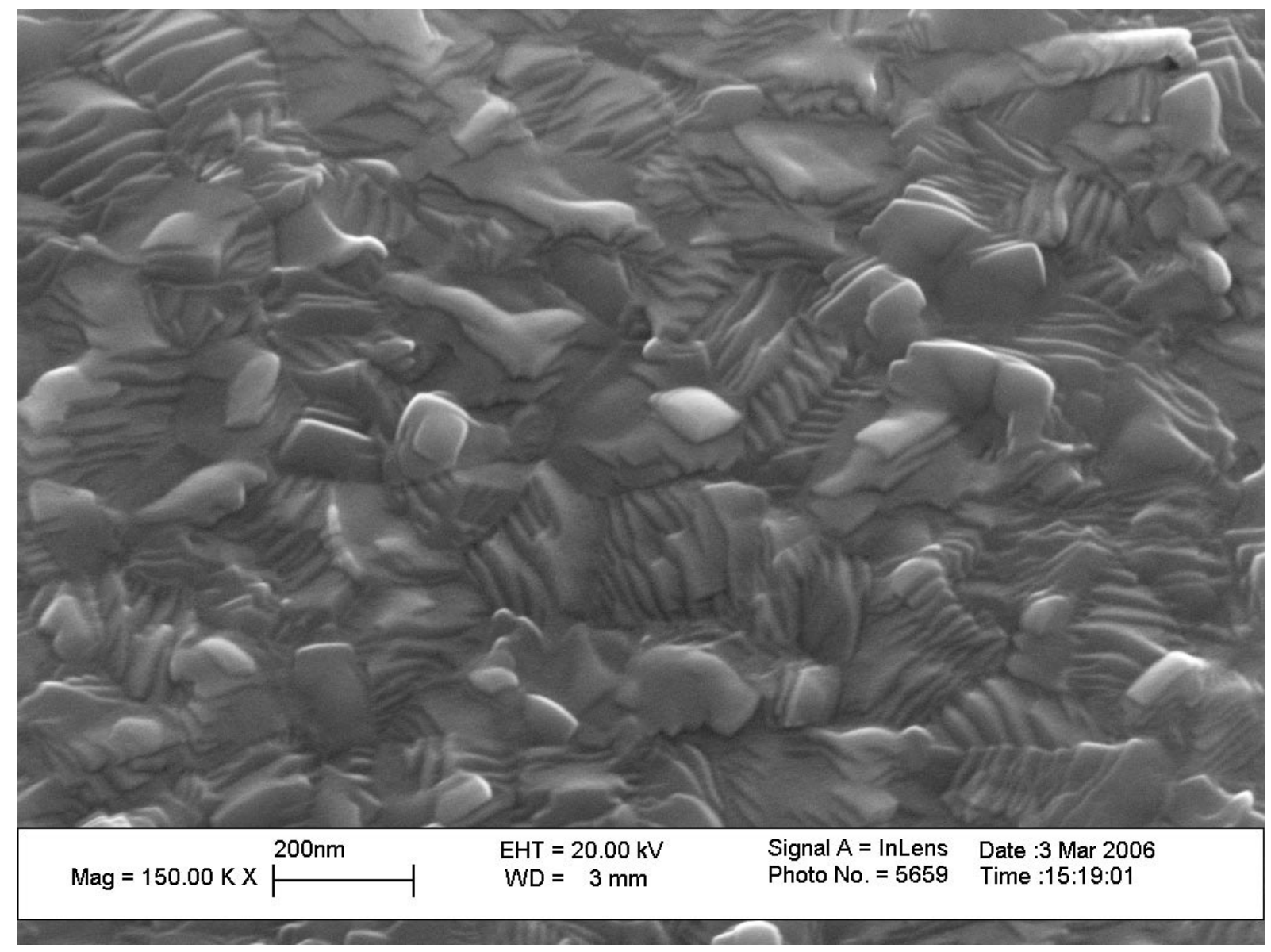

Figure 6c 